\title{
Структура каналов для считывающей электроники кремниевых детекторов
}

\author{
А. Воронин, к. $\Phi^{-м}$. н. $^{1}$
}

УДК 539.1.075 | ВАК 05.27.01

\begin{abstract}
Одна из особенностей считывающей электроники (СЭ) аппаратуры для экспериментов в области физики высоких энергий и космических лучей, электроники для некоторых других классов приборов, использующей кремниевые системы детектирования ионизирующих излучений, - многоканальность. Например, количество идентичных измерительных каналов СЭ установок для физики высоких энергий, каждый из которых подключен к одному сегменту детектора (стрипу или паду), может превышать 107. Специализированные интегральные микросхемы (СИМС), применяемые в СЭ, содержат, как правило, от 16 до 128 каналов. Рассмотрим основные структурные решения для СЭ и факторы, влияющие на выбор структурной схемы при реализации многоканальной детектирующей системы на основе кремниевых детекторов.
\end{abstract}

сновной задачей, для решения которой используется канал СЭ, является получение (измерение) достоверных характеристик импульсов одного сегмента детектора по напряжению и времени (в том числе в виде бинарной последовательности импульсов полезных сигналов). Применяемые в настоящее время структурные решения измерительных каналов в зависимости от технических требований - преимущественно аналогоцифровые, в состав которых могут входить головная часть, блоки аналоговой и цифровой обработки, АЦП, ЦАП.

Несмотря на многообразие схемных решений, можно выделить три основных типа каналов:

- последовательный;

- последовательно-параллельный;

- канал, образованный коммутацией отдельных блокОВ, входящих в СЭ

Канал первого типа, один из вариантов реализации
Под цифровыми блоками обработки сигнала понимаются блоки, осуществляющие цифровое преобразование сигнала в одном канале. Все более широкое применение технологии цифровой обработки связано с внедрением субмикронныхи наноэлектронных СИМ кремниевыхдетекторов, что значительно уменьшает размер вентиля на кристалле, потребляемую мощность и повышает рабочую частоту до несколькихгигагерц. Это позволяет применять аппаратные методы реализации сложных цифровых функций в многоканальной электронике, при этом обеспечиваются высокое быстродействие обработки сигналов и малые размеры цифровых устройств по сравнению с универсальными контроллерами. Общие для СЭ (СИМС) цифровые узлы, такие как управление, интерфейсы, общее преобразование данных в СЭ и другие подобные блоки, не входят в состав канала и в настоящей статье не рассматриваются, за исключением принципа работы арбитражной логики. которого показан на рис. 1, содержит цепь блоков, соединенных последовательно, и при необходимости может иметь обратные связи между блоками. Головная часть канала обеспечивает преобразование сигнала детектора в импульс напряжения, фильтрацию шумов и оптимизацию формы импульса, что позволяет выполнить дальнейшую обработку сигнала.

Научно-исследовательский институт ядерной физики им. Д. В. Скобельцина Мгу им. М.В. Ломоносова,

voronin@silab.sinp.msu.ru.

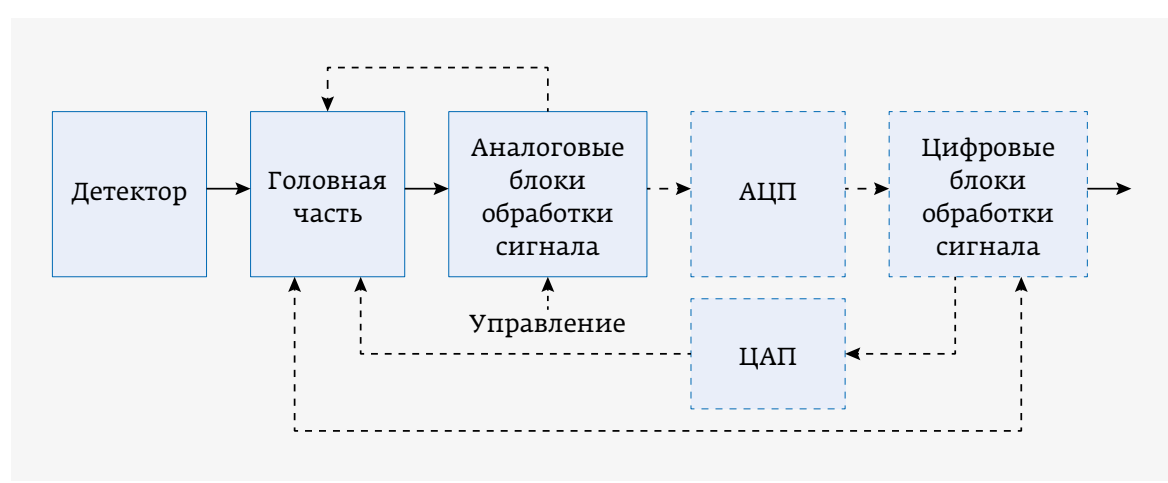

Рис. 1. Структура последовательного канала (штриховой линией обозначены блоки и связи, которые могут отсутствовать в реализации канала для конкретного проекта) 
Параметры аналоговых и цифровых блоков (коэффициент усиления, время формирования и др.) могут регулироваться с помощью внешних команд или с использованием адаптивных методов юстировки, что позволяет согласовать (улучшить) характеристики канала в целом.

Последовательно-параллельный канал (вариант реализации показан на рис. 2), имеющий общую головную часть, разделяется на несколько субканалов, каждый из которых оптимизирован для выполнения своей функции обработки и/или согласован с параметрами сегмента детектора этого канала. Например, канал может содержать субканал измерения амплитуды импульса и субканал $\mathrm{N}$ (см. рис. 2) для амплитудных измерений и определения времени (метки времени) или обеспечивать счетный режим событий (аналог счетчика Гейгера).

Между блоками субканалов возможны локальные связи, позволяющие упростить структуру субканалов. Например, оцифровка субканалов с разными временами формирования может осуществляться в АЦП только первого канала; для выделения метки времени события используется канал с минимальной длительностью формируемого импульса, а наличие полезного сигнала подтверждается субканалом, для которого характерен минимальный уровень шума.

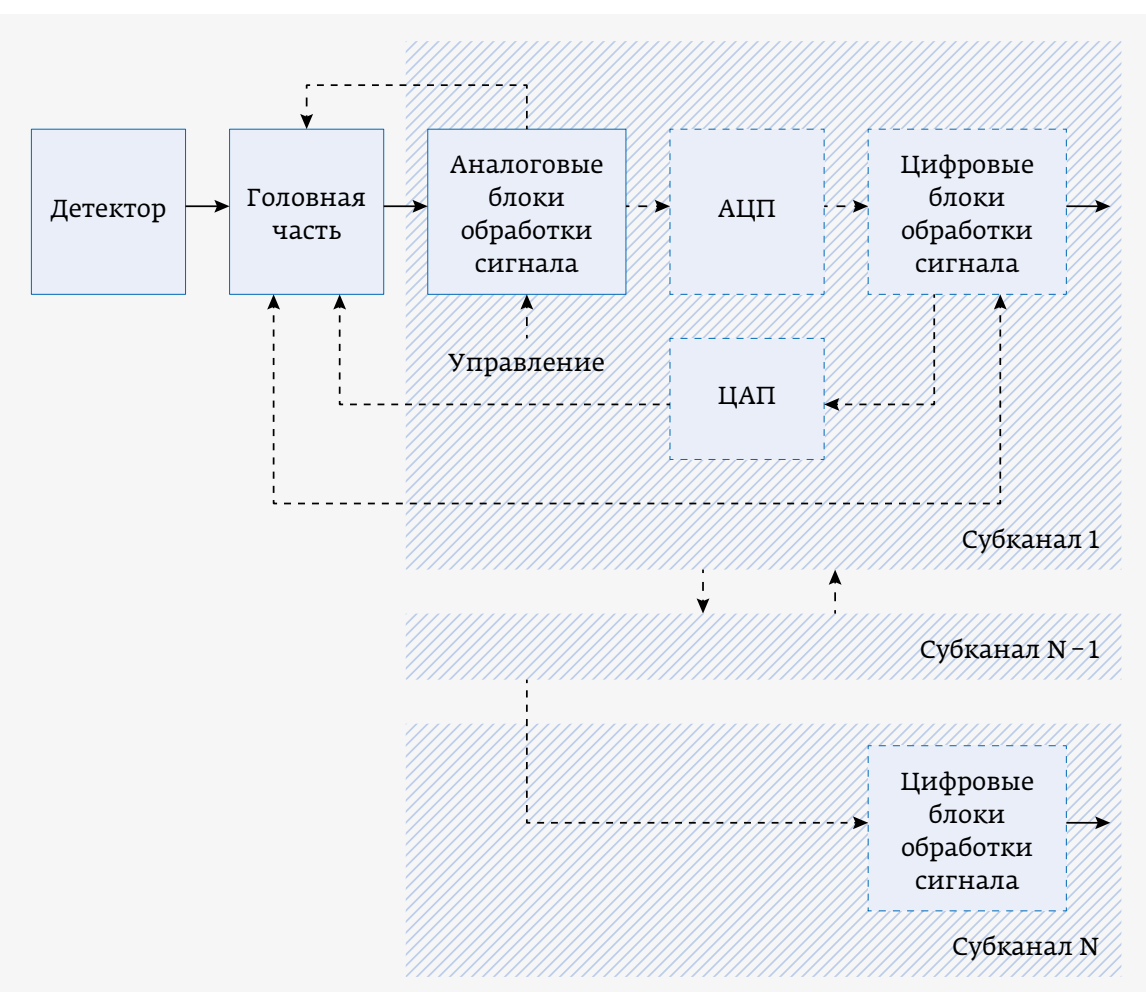

Рис. 2. Структура последовательно-параллельного канала (штриховой линией обозначены блоки и связи, которые могут отсутствовать в реализации канала для конкретного проекта)

Канал, образованный путем переключения входов и выходов элементов набора отдельных блоков (рис. 3), позволяет уменьшить количество однотипных устройств в многоканальной СЭ. Такая схема канала применима, если загрузка каналов невелика и / или время обработки для разных блоков значительно различается. Это относится как к аналоговой, так и цифровой частям СЭ. В данном

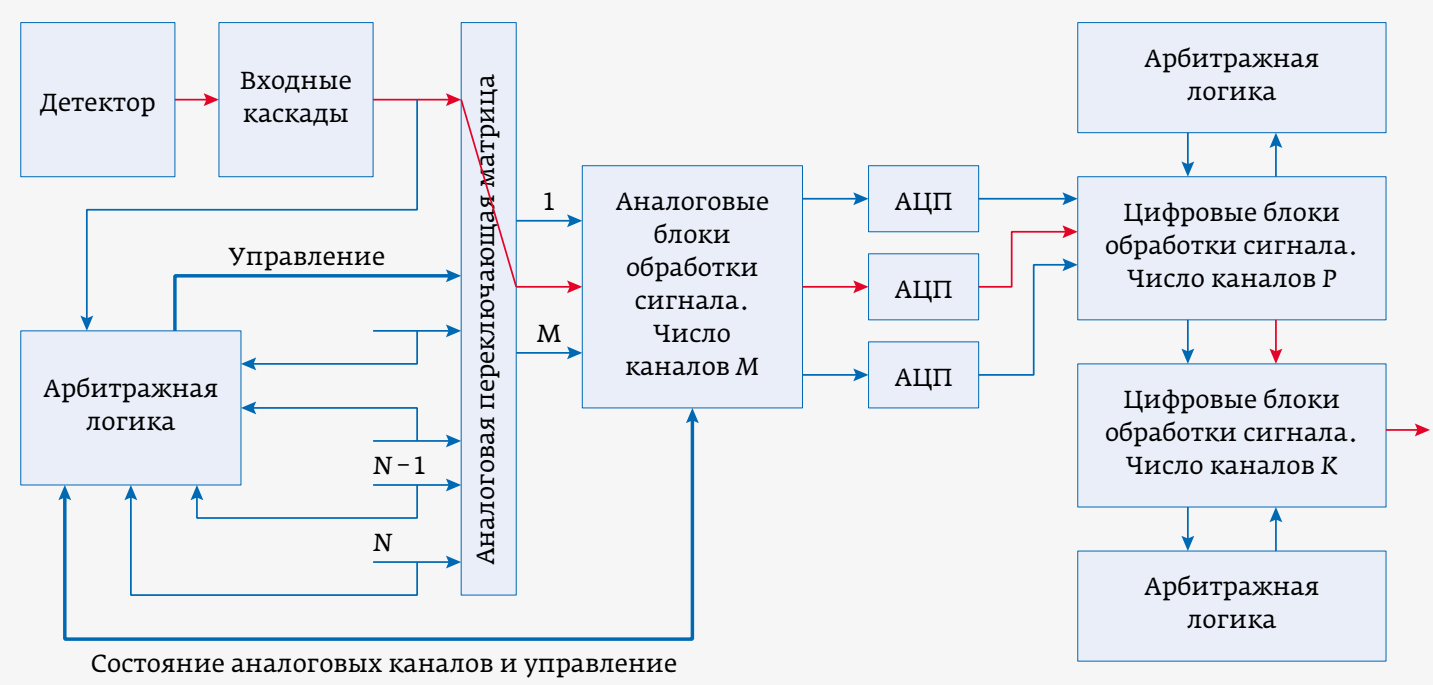

Рис. 3. Канал, образованный соединением устройств из набора отдельных укрупненных блоков (красными линиями выделено образование одного канала) 
случае канал делится на несколько укрупненных блоков, при этом в кристалле СИМС или СЭ создается набор аналогичных блоков, состоящий из субканалов каждого типа и содержащий необходимое количество устройств, которое должно обеспечивать обработку всех сигналов на входе при максимальной загрузке Сэ (СИМС). При этом число входов $N>M$ (где $M$ - число аналоговых субканалов), соответственно уменьшается количество АЦП и цифровых блоков.

Схема должна реализовать минимально допустимую вероятность пропуска полезного сигнала с учетом времени обработки в каналах, создаваемых по принципу переключения укрупненных блоков. Каждый входной каскад (число входов N) подключается к входу аналогового мультиплексора (матрицы), к другим входам которого подключаются входные каскады иных сегментов детекторов. Полный канал для одного сегмента формируется из свободных на данный момент блоков СЭ (на рис. 3 показан линиями красного цвета).

Дополнительная аналоговая переключающая матрица может быть расположена между аналоговыми блоками и АЦП. Арбитражная логика (схема управления) обеспечивает коммутацию блоков СЭ, создает один канал обработки для одного сегмента детектора и должна сохранять информацию о состоянии блоков СЭ ("свободен" или «занят») и коммутирующих устройств, а также формировать команды для переключения блоков (см. рис. 3). Арбитражная логика для цифровых блоков может быть проще, чем для аналоговых блоков, поскольку данные на выходе АЦП привязаны к тактовой частоте, в отличие от входных сигналов, случайно появляющихся на входе канала. Арбитражная логика содержит регистры состояния и решающее устройство, обеспечивает привязку данных к номеру входного канала и при необходимости привязку ко времени появления события.

Каждый отдельный укрупненный блок, показанный на рис. 1-3, состоит из функциональных узлов (функциональных блоков), обеспечивающих формирование, преобразование, фильтрацию шумов и измерение импульсов детекторов. Сигнал детектора - аналоговый, поэтому первый каскад Сэ выполняется как зарядочувствительный усилитель (зЧУ), или интегратор тока детектора, либо как преобразователь тока детектора в импульс напряжения. Косновным функциональным узлам канала кремниевого детектора относятся:

- фильтр-формирователь с бесконечной импульсной характеристикой (время-инвариантный шейпер) или конечной импульсной характеристикой (время-вариантный шейпер);

- компенсатор полюса Зчу, уменьшающий смещение базовой линии в канале;

- стабилизатор базовой линии, компенсирующий изменения постоянной составляющей, которая возникает при случайном во времени потоке импульсов детектора и нестабильности головного каскада;

- память;

- пиковый детектор;

- ЦАП и АЦП.

Кроме того, в состав функциональных блоков входят каскады усилителей, компараторы, ключи и мультиплексоры (переключающие матрицы), опорные источники напряжения, фильтры и другие каскады.

Функциональные блоки могут быть реализованы в аналоговом и цифровом виде, причем цифровая реализация узлов может быть выполнена как цифровое подобие аналоговых блоков или при помощи оригинальных цифровых методов.

Функции обработки сигнала детектора осуществляются в канале по запускающему триггеру (команде), что позволяет существенно уменьшить объем информации, которую должна обработать СЭ. В зависимости от вида триггера применяют разные структурные схемы (различный набор функциональных блоков) для построения канала. Существуют два основных метода триггирования канала: по внешнему триггеру и самотриггирование канала.

\section{КАНАЛ С ВНЕШНИМ ТРИГГЕРОМ}

Принцип работы канала с внешним триггером рассмотрим на примере последовательного канала с аналоговой реализацией основных блоков (рис. 4). Описание аналоговой части подобного канала без триггера и входящих в него узлов, а также схема подключения триггера приведены в [1, 4]. Часть блоков канала, таких как стабилизатор базовой линии, формирователь, уменьшающий длительность импульса и снижающий вероятность

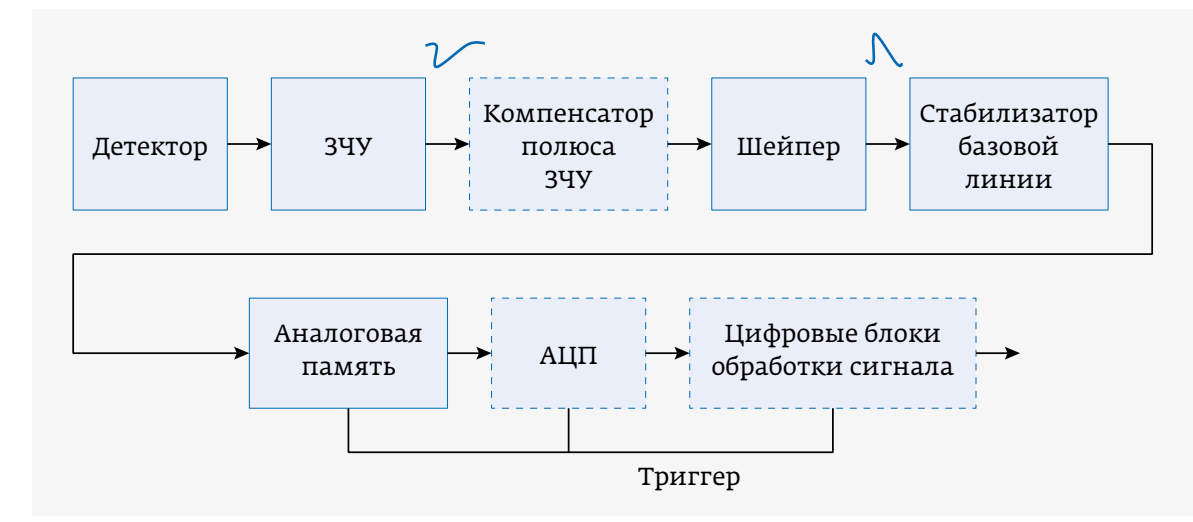

Рис. 4. Пример канала с внешним триггером 


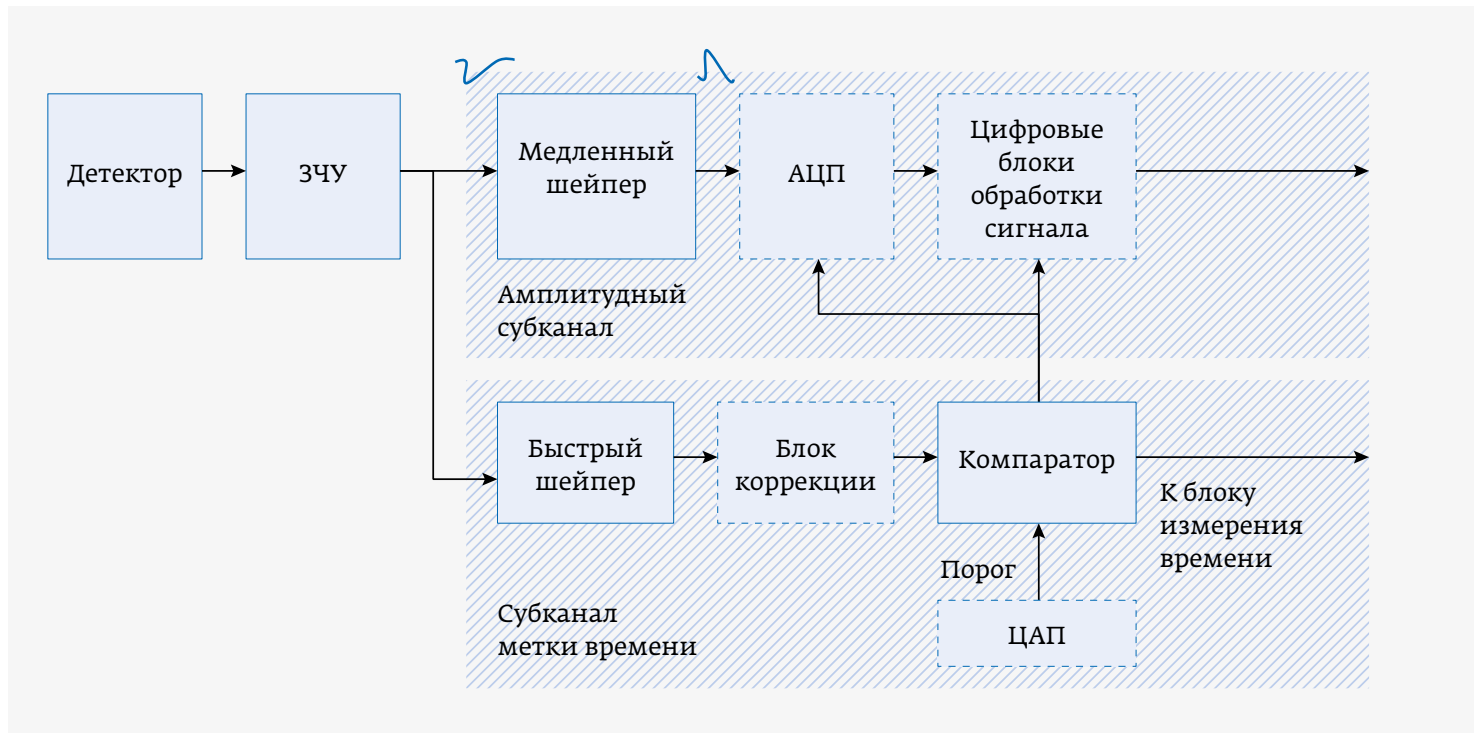

Рис. 5.

Пример канала с самотриггированием наложений соседних событий, или дополнительный пиковый детектор, можно реализовать в цифровом виде.

При внешней триггерной системе структура канала и его реализация существенно упрощаются, однако основной недостаток внешнего триггера - низкое быстродействие. Это связано с тем, что внешняя триггерная система обрабатывает большое количество каналов, и необходимо дополнительное время на принятие решения о выработке триггера - не менее сотен наносекунд. Поэтому аналоговая память (блок задержки) - ключевой блок для правильной работы канала по внешнему триггеру. Аналоговая память может служить также пиковым детектором, если триггер подать во время достижения максимума импульсом на выходе шейпера.

\section{КАНАЛ С САМОТРИГГИРОВАНИЕМ}

Канал с самотриггированием (то есть решение о наличии сигнала принимается в самом канале, в английском варианте data driven) выполняется, как правило, по последовательно-параллельной схеме (см. рис. 2, 5). Для этого вводится субканал с шейпером, для которого выбирается минимально возможное время формирования, которое для кремниевых детекторов составляет десятки наносекунд. Быстрое время формирования минимизирует время определения события и позволяет его фиксировать в блоке измерения времени, привязывая кадресу канала для дальнейшей локализации данных о событии со всех каналов установки.

Возможно введение блока коррекции, который стабилизирует или компенсирует разброс задержки срабатывания компаратора, возникающий за счет конечности времени фронта быстрого шейпера. Схема с самотриггированием более быстродействующая по сравнению со схемой с внешним триггером, поскольку решение о наличии сигнала принимается в головных каскадах одного канала.
Субканал метки времени запускает также обработку сигнала в основном амплитудном канале, время формирования которого больше, чем в быстром канале, что позволяет снизить шум и пренебречь задержкой быстрого канала. Столь простая схема субканала метки времени дает возможность предположить наличие ошибок при ее работе.

Известно, что ошибки вносят два основных фактора шум и статистический разброс величин ионизационных потерь в кремнии и, как следствие, разброс амплитуды сигнала в кремниевом детекторе. Для релятивистских частиц амплитудный спектр сигнала приближенно можно описать кривой, аналогичной распределению Ландау (рис. 6).

На рис. 6, показывающем пересечение амплитудных спектров сигнала и шумов, видно, что практически невозможно установить порог, полностью исключив шум. Ошибки при определении метки времени можно классифицировать, используя понятие выбросов шумов, то есть пересечение шумами порога компаратора:

- ложный сигнал (ложная тревога) или потеря полезного сигнала;

- ошибка в измерении времени события.

Кроме того, на входе быстрого шейпера шумовой выброс может соединить близко расположенные сигналы в один (выброс “снизу-вверх») или разделить один сигнал на несколько (выброс “сверху-вниз»).

Амплитудный спектр с обрезанным шумом, полученный в процессе эксперимента СВД-2, представлен на рис. 7.

Суммарная длительность импульсов в канале метки времени кремниевого детектора составляет 20-40 нс/ импульс, что не превышает нескольких процентов даже при высокой загрузке канала (например, при частоте до 1 МГц). Можно утверждать, что основным источником ошибки (ложной тревоги) в канале являются 


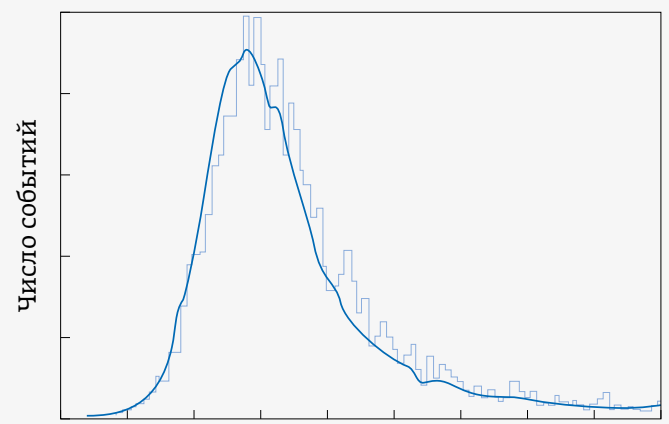

Амплитуда импульсов

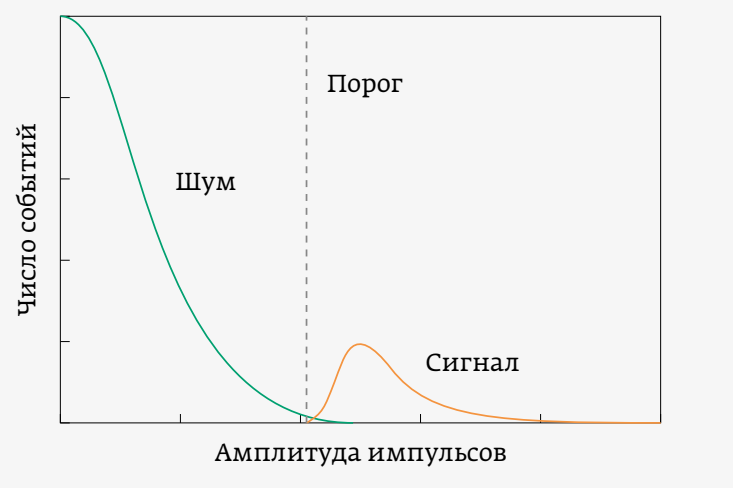

Рис. 6.

Вид распределения Ландау (слева) и амплитудный спектр сигнала и шума (справа) шумовые выбросы в отсутствии сигнала. Для нормальных стационарных шумовых процессов среднее число шумовых выбросов “снизу-вверх" $\mathrm{N}_{1}$ (а также “сверху-вниз» $\mathrm{N}_{2}$ ) в единицу времени в общем виде равно $[2,3]$ :

$$
\mathrm{N}_{1}=\frac{1}{2 \pi} \sqrt{-\mathrm{R}(0)^{\prime \prime}} \cdot e^{-0,5 \gamma^{2}}
$$

где R(0)" - вторая производная от корреляционного коэф-

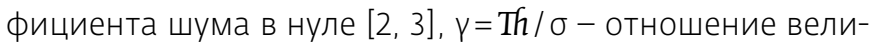
чины порога Тh к среднеквадратическому отклонению б, взвешенного в полосе шейпера;

$$
R(0)^{\prime \prime}=-\frac{1}{2 \pi \sigma^{2}} \int_{-\infty}^{\infty}(2 \pi f)^{2} \cdot S(2 \pi f) \cdot d(2 \pi f),
$$

где $S(2 \pi f)$ - спектральная плотность шума, $f$ - циклическая частота.

В нашем случае на входе канала преобладающей шумовой составляющей является "белый" шум со спектральной плотностью $\mathrm{N}_{0}$, поэтому $S(2 \pi f)$ можно выразить следующим образом:

$$
S(2 \pi f)=N_{0}\left|k_{n}(2 \pi f)\right|,
$$

где $\left|k_{n}(2 \pi f)\right|-$ модуль коэффициента передачи для шумов канала метки времени с зчу.

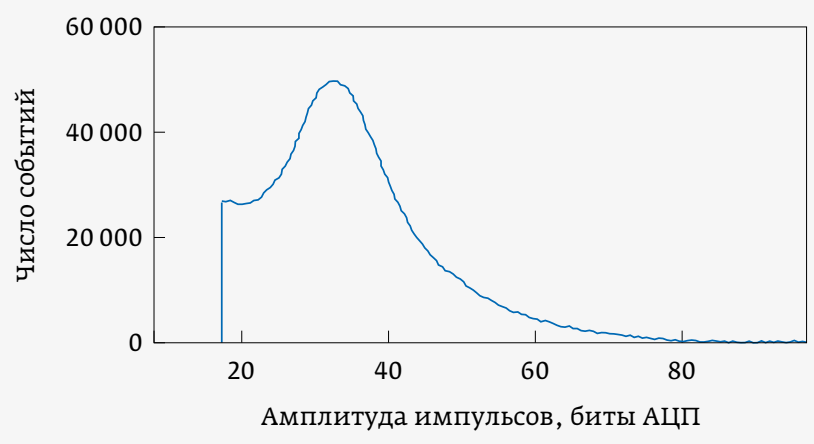

Рис. 7. Амплитудный спектр канала эксперимента СВД-2
Выражения для $\left|k_{n}(2 \pi f)\right|$ достаточно просто получить, используя работы [1, 3, 5], с учетом различных видов шейперов $\left(k_{n S H}\right)$ (коэффициент передачи $\left.k_{n}(2 \pi f)=k_{n з ч y} \cdot k_{n S H}\right)$.

В канале метки времени используются в основном CR-RC-шейперы, образующие полосовой фильтр и состоящие из последовательно соединенных дифференцирующих и интегрирующих каскадов. Отклик такого шейпера представляет собой импульс псевдогауссовой формы, а форма амплитудного спектра шума на выходе фильтра близка к гауссовой форме. В работе [2] показано, что количество выбросов пропорционально полосе спектральной плотности полосового фильтра $k \cdot \Delta f$. Коэффициент k определяет поведение огибающей $\left|k_{n}(2 \pi f)\right|$. Поскольку ложный запуск канала метки времени осуществляется выбросами "снизу-вверх", для оценки k полосового импульса псевдогауссовой формы можно предположить, что ложный запуск дает половина числа переходов шумов через порог в полосе $\Delta f$ и $k \approx 0,5$. Оценить среднее число выбросов $N_{1}$ в единицу времени и соответственно число ложных запусков в канале можно по следующей формуле:

$$
N_{1} \cong 0,5 \cdot \Delta f \cdot e^{-0,5 \gamma^{2}},
$$

где $\Delta f$ - полоса спектральной плотности по уровню 0,5 по мощности (0,707 по напряжению), которую можно получить, преобразовав $\left|k_{n}(2 \pi f)\right|$, используя [3].

Более точное значение $\mathrm{N}_{1}$ можно рассчитать, применив выражения (1) - (3); например, для идеального гауссового спектра $k=0,42$.

Напряжение порога выбирают исходя из $\gamma=T h / \sigma$ так, чтобы количество ложных срабатываний было пренебрежимо мало по сравнению со средней частотой загрузки канала $f_{\text {о. }}$ Очевидно, что величина порога должна быть существенно меньше максимального сигнала детектора Umipс для минимально ионизующей частицы (рис. 6, 7) на входе компаратора и находиться в зоне пересечения амплитудных спектров шумов и сигнала.

Для кремниевого детектора наиболее вероятный (максимальный) выходной сигнал на единицу толщины детектора составляет заряд Qmip / мкм =12·10-18 K/ мкм. 


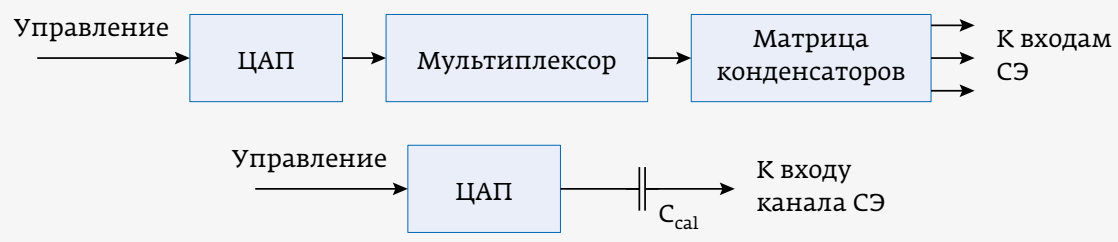

Рис. 8. Структура тестового канала: вверху - для группы измерительных каналов; внизу - для индивидуального измерительного канала

Преобразуем Qmip в напряжение: Umip=Qmip / Ceq. Емкость Сеq - это сумма емкости детектора и всех паразитных емкостей, подключенных к входу СЭ. Умножив коэффициент передачи сигнала $k_{\Sigma^{\prime}}$ рассчитанный используя [3], на Umip, получим амплитуду импульса на входе компаратора Umipc $=U$ mip $\cdot k_{\Sigma}$ для конкретной схемы канала с коэффициентом передачи $k_{\Sigma}$

Обычно на выходе шейпера канала с кремниевым детектором отношение сигнал-шум SNR=Qmipsh $/ \sigma_{\text {sh }}$ находится в пределах 5-15, соответственно порог не может превышать несколько $\sigma$. Необходимо найти компромисс между максимальным быстродействием и отношением сигнал-шум в канале, чтобы число ложных срабатываний было допустимо минимальным, а эффективность регистрации (пропуск полезного события) - максимальной. Это достигается, в частности, юстировкой аппаратуры (экспериментальной регулировкой порога).

Следует отметить, что экспонента в выражениях для выбросов быстро спадает из-за $\gamma^{2}$ (1), что обеспечивает эффективность регистрации на уровне выше $90 \%$. Оценить эффективность регистрации полезного сигнала по отношению к выбросам можно с использованием данных амплитудного субканала (см. рис. 5), поскольку SNR в этом канале выше, чем в канале метки времени. Канал, показанный на рис. 3, также является самотриггирующимся.

Кроме измерительных каналов (каналов регистрации), в СЭ (СИМС) для кремниевых детекторов вводятся калибровочные каналы, позволяющие контролировать работоспособность аппаратуры.

\section{КАЛИБРОВОЧНЫЙ КАНАЛ}

Калибровочный (тестовый) канал в СЭ (СИМС) выполняет две функции: проверку работоспособности каналов СЭ и создание информационной базы для коррекции данных при деградации СЭ, например в космических экспериментах.

Калибровочный канал может быть одним для группы измерительных каналов (рис. 8). В этом случае можно выбирать только номера каналов с помощью мультиплексора. Тестовые сигналы при этом будут одной и той же амплитуды.
Калибровочный канал также может быть реализован в каждом измерительном канале, в этом случае можно создать любую комбинацию тестовых воздействий с разными амплитудами (см. рис. 8).

Калибровочный канал не может полностью имитировать пролет частицы через кремниевый детектор, поскольку он подключен непосредственно к входам СЭ и не учитывает детекторную часть измерительного канала, в частности задержки. Форма тестового сигнала генерируется ЦАП, как правило, в виде прямоугольного импульса длительностью $\Delta$ t, что не соответствует в полной мере форме сигнала детектора. Корреляцию между калибровочной системой и сигналом от частицы можно установить при помощи эксперимента с источником соответствующего излучения или лазера.

Калибровочная емкость $C_{c a l}$ выбирается из условия $\mathrm{C}_{\text {cal }}<<\mathrm{C}_{d}\left(\mathrm{C}_{d}-\right.$ емкость детектора), чтобы уменьшить паразитную емкость входа. ЦАП может иметь выход по напряжению $\mathrm{U}_{\mathrm{dac}}$ тогда входной тестовый заряд $\mathrm{Q}_{\mathrm{t}}=\mathrm{U}_{\mathrm{dac}} \cdot \mathrm{C}_{\mathrm{cal}}$, или по однополярному току $\mathrm{I}_{\mathrm{t}}: \mathrm{Q}_{\mathrm{t}}=\mathrm{I}_{\mathrm{t}} \cdot \Delta t$.

\section{ЗАКЛЮЧЕНИЕ}

В статье в обобщенном виде рассмотрены структурные схемы каналов многоканальной электроники для регистрации и тестирования приборов, использующих в качестве датчика кремниевые детекторы, а также вопросы, связанные с подключением внешнего триггера, и особенности триггера, расположенного в самом канале. Кроме того, кратко описана структура калибровочного (тестового) канала для проверки работоспособности остальных (рабочих) каналов. Положения этой статьи предполагается использовать при реализации гранта РФФИ № 18-02-40047 «Система скоростного потокового чтения данных с широко-апертурной кремниевой трековой системы установки NICA-BM@N».

\section{ЛИТЕРАТУРА}

1. Spieler H. Semiconductor Detector Systems // John Wiley Oxford University Press, New York U. S.A., 2005

2. Тихонов В.И. Выбросы случайных процессов // Успехи физических наук. 1962. Т. LXXVII. Вып. 4.

3. Баскаков С. И. Радиотехнические цепи и сигналы / 3-е изд. - М.: Высшая школа, 2000.

4. Spieler H. Electronics and data acquisition // Nuclear Instruments and Methods, 2012, V. A666, P. 197-222.

5. Grybos P. Front-end Electronics for Multichannel Semiconductor Detector Systems. // Institute of Electronic Systems, Warsaw University of Technology, Warsaw, 2010. 